\title{
Erectile dysfunction with elevated serum testosterone
}

\author{
L D Ranasinghe ${ }^{1}$, K D Liyanarachchi ${ }^{1}$, N P Somasundaram ${ }^{1}$ \\ Sri Lanka Journal of Diabetes, Endocrinology and Metabolism 2015; 5: 100-102
}

\begin{abstract}
Symptoms of testosterone deficiency with elevated testosterone levels are rare and could occur with elevated sex hormone binding globulin (SHBG) levels. Here, we report a 59 year old patient presenting with erectile dysfunction (ED) with diminished libido for 4 months. He developed these symptoms 6 months after starting anti tuberculosis treatment (AAT). He had elevated total testosterone concentration along with raised SHBG. However, his free testosterone was normal. Repeated total testosterone level 2 months after completion of ATT was normal indicating normalization of SHBG levels.
\end{abstract}

Key words: Erectile Dysfunction, SHBG, ATT.

\section{Introduction}

ED is defined as persistent or recurrent inability to achieve and maintain penile erection of sufficient rigidity to permit satisfactory sexual activity for at least 3 months (1). The etiology of erectile dysfunction is multi factorial and infections such as tuberculosis (TB) could also lead to ED due to the hypogonadism with the related involvement of the pituitary and testicules. Inspite of having normal genitourinary system, patients with pulmonary TB tend to have deterioration of all components of copulatory act, from sexual desire to orgasm, which improve significantly with effective ATT (2). On the other hand, ATT is also known to cause ED, which could resolve with the discontinuation of treatment (3).

\section{Case report}

A 59 year old male who had no relationship problems presented with ED for 4 months. He had decreased libido with absent morning erections. He did not have symptoms of depression though he had psychological distress related to ED. He was diagnosed to have tuberculus meningitis 10 months ago when he presented with loss of consciousness associated with headache and a febrile illness. He was started on ATT and he has been on isoniazid, rifampicin and pyridoxine during the last 10 months. He tolerated anti TB therapy fairly well and there were no episodes of ATT induced hepatitis. He also had hypertension and benign prostatic hyperplasia for which he was on amlodipine and tamsulosin. He was otherwise well. He denied use of steroids except at the initial 2 months of ATT or recreational drugs. He had 3 children and was a nonsmoker and a social drinker. Examination revealed a well virilized male with a normal testicular consistency and size $(20 \mathrm{ml})$. Rest of the examination was unremarkable.

Biochemical evaluation revealed repeatedly raised 9am total testosterone levels of 64.3 and $57 \mathrm{nmol} / 1$ (9.738.14). Free testosterone level performed using enzyme linked immunosorbent assay (ELISA) was $15.84 \mathrm{pg} / \mathrm{ml}$ (5.5 -42), which was normal. He had a raised SHBG level of $112 \mathrm{nmol} / 1$ (13- 71), which probably explaining the reason for raised total testosterone. Follicle stimulating hormone (FSH) was slightly raised (10.5 U/L) while, luteinizing hormone ( $\mathrm{LH})$ was normal (6 U/L). Other anterior pituitary hormones including 9am cortisol, thyroid stimulating hormone (TSH), $\mathrm{FT}_{4}$ and prolactin were normal. His serum albumin was $43 \mathrm{~g} / \mathrm{L}$ and other hematological and biochemical markers including liver profile were normal.

Ultrasound scan of the scrotum showed normal testicular size, echogenicity and vascularity on both sides. There was no testicular calcification. An epididymis cyst was noted on right side ( $5 \mathrm{~mm}$ size). Minimal scrotal effusions were also noted bilaterally.

The patient was explained the possible hormone derangement due to ATT. He was reassured and informed the possibility of reversibility of sexual function after discontinuation of ATT once treatment is completed. As expected, his total testosterone level got normalized and came down to $22.09 \mathrm{nmol} / 1$ (9.7- 38.14) 2 months after stopping ATT, indicating that raised SHBG in this patient is most likely due to anti TB medication. 


\section{Discussion}

Certain drugs are known to cause elevation of testosterone levels and rifampicin has been one of those drugs that could increase the level of testosterone $(4,5)$. Brodie, et al has demonstrated that 2 week rifampicin therapy at a dose of $600 \mathrm{mg}$ per day increased the level of testosterone and estradiol in males with testosterone returning to normal range within 2 weeks after stopping treatment (4). SHBG levels were also significantly increased after 2 weeks of rifampicin treatment and then fell down to normal level after discontinuation of drug. However, he could not demonstrate a significant correlation between the changes in testosterone and SHBG levels. Raised levels of total testosterone, (and SHBG) while on isoniazid and rifampicin, came down to normal range after stopping these drugs indicate that either isoniazid or rifampicin was responsible for this hormonal change in our index patient. Similar to the above study, rifampicin induced SHBG over production could have been the most likely explanation for the rise in total testosterone concentration in our patient.

Rifampicin induces cytochrome P450 mediated monooxygenase activity (cholesterol side-chain cleavage and 17 alpha-hydroxylase/C17-20 lyase) is required for testosterone biosynthesis in leydig cells (6). Increased biosynthesis of testosterone may occur in patients on rifampicin due to this cytochrome $\mathrm{P} 450$ induction. Due to the associated elevation of SHBG, most of the testosterone produced with the activation of cytochrome $\mathrm{P} 450$ will get bound to SHBG leading to reduction in biologically active free testosterone level. This reduction in bioavailable testosterone due to high SHBG leads to clinically significant problems such as erectile dysfunction. Therefore, it is important to check free testosterone level in cases where changes in SHBG are suspected (7).

Most of the freely available assays for free or bioavailable testosterone measurements are not very reliable and should be performed in a reliable reference laboratory (7). Free testosterone level can be measured accurately by equilibrium dialysis or can be calculated using total testosterone, SHBG and albumin (8). The calculated free testosterone concentrations depends on the quality of total testosterone and SHBG assays and differ systematically from those measured by equilibrium dialysis (9). Our patient had symptoms of testosterone deficiency despite high total testosterone levels and normal free testosterone levels. The assay that was used to measure free testosterone was not the ideal and could have given a misleading value.

Anabolic steroids, thyrotoxicosis, HIV infection, malnutrition (low $\mathrm{Mg}, \mathrm{Zn}$ ) and liver disease are known to cause SHBG elevation. Anticonvulsants notably phenytoin and phenobarbitone can also increase SHBG and total testosterone levels $(10,11)$. As a result, free testosterone may reduce and could be the reason for ED in epileptic patients. Clomiphene citrate causes an increase of SHBG and total testosterone concentration, probably due to the elevation of estradiol levels (12). Total testosterone level can be significantly elevated in patients with thyrotoxicosis and this is also due to excessive production of SHBG. They can develop ED due to low free testosterone, which improves with treatment of thyrotoxicosis. Therefore, specific treatment of ED with selective phosphodiesterase-5 inhibitor should be postponed for at least 6 months after restoration of euthyroidism in these patients (13).

Variety of drugs and medical conditions could alter the levels of SHBG and total testosterone level should be interpreted cautiously in the presence of above conditions. Anti-tuberculosis medication, rifampicin is one of these drugs.

\section{References}

1. Bella AJ, Lee JC, Carrier S, Bénard F, Brock GB. 2015 CUA Practice guidelines for erectile dysfunction. Can Urol Assoc J 2015; 91(2): 23-9.

2. Kulchavenya E, Scherban M, Brizhatyuk E, Osadchiy A. Sexual dysfunction in male patients with pulmonary tuberculosis. J Microbiol Infect Dis 2012; 2(3): 124-6.

3. Gupta KB, Kumar V, Vishvkarma S, Shandily R. Lung India 2011 ; 28(1): 60-1.

4. Brodie MJ, Boobis AR, Gill M, Mashiter K. Does rifampicin increase serum levels of testosterone and oestradiol by inducing sex hormone binding globulin capacity? Br J Clin Pharmacol 1981; 12(3): 431-3.

5. Nocke-Finck L. and Breuer H. Wirkung von Rifampicin und Streptomycin auf Konzentrationen von Testosteron und Cortisol im Blut von Mannern. J. clin chem. Biochem 1980; 18: 897-9.

6. Ohnhaus E, Park BK. Measurement of 6-, fhydroxycortisol excretion as an in vivo parameter in the clinical assessment of the microsomal enzyme-inducing capacity of antipyrine, phenobarbitone and rifampicin. Eur. J clin Pharmac 1979; 15: $139-45$.

7. The endocrine society's clinical guideline. Testosterone therapy in adult men with androgen deficiency syndromes: An endocrine society clinical practice guidelines. J Clin Endocrinol Metab 2010; 95(6): 2536-59.

8. Vermeulen A, Verdonck L, Kaufman JM. A critical evaluation of simple methods for the estimation of free testosterone in serum. J Clin Endocrinol Metab 1999; 84: 3666-72.

9. Sartorius G, Ly LP, Sikaris K, McLachlan R, Handelsman DJ. Predictive accuracy and sources of variability in calculated free testosterone estimates. Ann Clin Biochem 2009; 46: 137-43. 
10. Barragry JM, Makin HL, Trafford DJ, Scott DF. Effect of anticonvulsants on plasma testosterone and sex hormone binding globulin levels. JNeurol Neurosurg Psychiatry 1978; 41(10): 913-4.

11. Smaldone M, Sukkarieh T, Reda A, Khan A. Epilepsy and erectile dysfunction: a review. Seizure 2004; 13(7): 453-9.

12. Adamopoulos DA, Vassilopoulos P, Kapolla N,
Kontogeorgos L. The effect of clomiphene citrate on sex hormone binding globulin in normospermic and oligozoospermic men. Int J Androl 1981; 4(6): 639-45.

13. Gerasimos E. Krassas, Kostas Tziomalos, Fotini Papadopoulou, Nikolaos Pontikides, and Petros Perros. Erectile Dysfunction in Patients with Hyper- and Hypothyroidism: How Common and Should We Treat? J Clin Endocrinol Metab 2008; 93: 1815-9. 